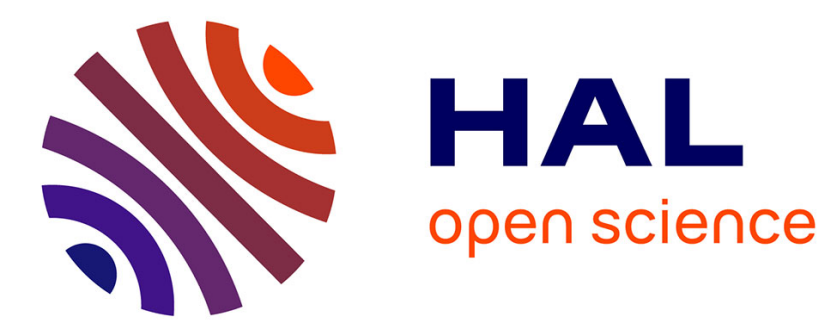

\title{
Stabilization of nonlinear systems subject to uncertainties and actuator saturation
}

Souad Bezzaoucha, Benoît Marx, Didier Maquin, José Ragot

\section{To cite this version:}

Souad Bezzaoucha, Benoît Marx, Didier Maquin, José Ragot. Stabilization of nonlinear systems subject to uncertainties and actuator saturation. American Control Conference, ACC 2013, Jun 2013, Washington, United States. pp.CDROM. hal-00782928

\section{HAL Id: hal-00782928 \\ https://hal.science/hal-00782928}

Submitted on 8 Apr 2014

HAL is a multi-disciplinary open access archive for the deposit and dissemination of scientific research documents, whether they are published or not. The documents may come from teaching and research institutions in France or abroad, or from public or private research centers.
L'archive ouverte pluridisciplinaire HAL, est destinée au dépôt et à la diffusion de documents scientifiques de niveau recherche, publiés ou non, émanant des établissements d'enseignement et de recherche français ou étrangers, des laboratoires publics ou privés. 


\title{
Stabilization of Nonlinear Systems Subject to Uncertainties and Actuator Saturation
}

\author{
Souad Bezzaoucha, Benoît Marx, Didier Maquin, José Ragot
}

\begin{abstract}
This paper deals with nonlinear system control with input saturation and parametric uncertainties. The considered nonlinear systems are represented by Takagi-Sugeno models. The proposed controller is a parallel distributed compensation state feedback. Stabilization conditions are derived with the Lyapunov method and expressed as an optimization problem under linear matrix inequality (LMI) constraints. The obtained controller gains depend on the saturation limits. The descriptor approach for modelling is also applied to reduce the number of LMI. An academic example is presented with a comparison between the proposed approach and a conventional controller.
\end{abstract}

Index Terms - Nonlinear system, uncertain system, TakagiSugeno model, actuator saturation, linear matrix inequality, descriptor system.

\section{INTRODUCTION}

In this paper, uncertain nonlinear systems represented by Takagi-Sugeno (T-S) models with actuator saturation constraints are considered. In the existing literature, several approaches are proposed to deal with the saturation problem. One can find the anti-windup controller, a two-step approach in which a nominal linear controller is first constructed by ignoring actuator saturation. Once this controller is designed, usually a so called anti-windup compensator is added to handle the saturation constraints. A typical anti-windup scheme consists in augmenting the nominal pre-designed linear controller with a compensator based on the discrepancy between unsaturated and saturated control signals fed to the plant (see [7] and [6] for more details).

Actuator saturation is also dealt with by designing low gain control laws, which for a given bound on the initial conditions, avoid the saturation limits ([5], [11] and the references therein).

Another method is proposed in [2] where the T-S modelling approach is used to analyze the domain of attraction of nonlinear systems with actuator saturation. In [9], polytopic models are also used to represent the saturated closed-loop system for the synthesis of linear control systems and several conditions to ensure the local asymptotic stability of the closed-loop system are derived in the form of Bilinear or Linear Matrix Inequalities (BMI) or LMI. However, these polytopic differential inclusions only locally represent the saturated system.

In the present paper, the input saturation is straightly taken into account in the controller design process. For that, the T-S

\footnotetext{
The authors are with Centre de Recherche en Automatique de Nancy (CRAN), Université de Lorraine, CNRS, 2 avenue de la Forêt de Haye F-54516 Vandoeuvre-les-Nancy, France. firstname. name@univ-lorraine.fr
}

formalism is used to represent the nonlinear behaviour of the saturated actuator and a Parallel Distributed Compensation (PDC) method is used to design a state feedback controller for uncertain nonlinear systems. Stabilization conditions are derived with the Lyapunov method and expressed as LMI. However, it is important to highlight a crucial difference with respect to the previous cited method, in the proposed approach, the T-S model of the saturation is valid in the whole state space and represents the nonlinear actuator behaviour.

The paper is organized as follows: section II introduces the Takagi-Sugeno structure for modelling, some preliminary results and mathematical notations. It is followed by the representation of the nonlinear saturation by a T-S structure in section III. In section IV a state feedback control law depending on the saturation bounds is designed. In section $\mathrm{V}$, the descriptor approach is applied in order to reduce the number of LMI to solve. A numerical example and some simulation results are given in section VI. Conclusions and future works are detailed in section VII.

\section{PRELIMINARIES}

\section{A. Takagi-Sugeno structure for modeling}

The T-S modeling allows to represent the behavior of nonlinear systems by the interpolation of a set of linear submodels. Each submodel contributes to the global behavior of the nonlinear system through a weighting function $\mu_{i}(\xi(t))$ [8]. The T-S structure is given by

$$
\left\{\begin{aligned}
\dot{x}(t) & =\sum_{i=1}^{n} \mu_{i}(\xi(t))\left(A_{i} x(t)+B_{i} u(t)\right) \\
y(t) & =\sum_{i=1}^{n} \mu_{i}(\xi(t))\left(C_{i} x(t)+D_{i} u(t)\right)
\end{aligned}\right.
$$

where $x(t) \in \mathbb{R}^{n_{x}}$ is the system state, $u(t) \in \mathbb{R}^{n_{u}}$ is the control input and $y(t) \in \mathbb{R}^{m}$ the system output. $\xi(t) \in \mathbb{R}^{q}$ is the decision variable assumed to be measurable (as the system output or measurable states) or known (as the system input). The weighting functions $\mu_{i}(\xi(t))$ of the $n$ submodels satisfy the convex sum property

$$
\left\{\begin{array}{l}
\sum_{i=1}^{n} \mu_{i}(\xi(t))=1 \\
0 \leq \mu_{i}(\xi(t)) \leq 1, \quad i=1, \ldots, n
\end{array}\right.
$$

In the remaining of the paper, the three following lemmas are used. 
Lemma 1: Consider two matrices $X$ and $Y$ with appropriate dimensions, $\Sigma$ and $G$ symmetric positive definite matrices. The following property is verified

$$
-X^{T} \Sigma X-Y^{T} \Sigma^{-1} Y \leq X^{T} Y+Y^{T} X \leq X^{T} G X+Y^{T} G^{-1} Y
$$

Lemma 2: (Congruence) Consider two matrices $X$ and $Y$. If $X$ is positive (resp. negative) definite and if $Y$ is a full column rank matrix, then the matrix $Y X Y^{T}$ is positive (resp. negative) definite.

Lemma 3: Consider three matrices $X, Y$ and $\Sigma(t)$ with $\Sigma^{T}(t) \Sigma(t) \leq I$. For any positive scalar $\lambda$

$$
X^{T} \Sigma(t) Y+Y^{T} \Sigma^{T}(t) X \leq \lambda X^{T} X+\lambda^{-1} Y^{T} Y
$$

\section{B. Mathematical notations}

The following notations are used throughout the paper.

A bloc diagonal matrix with the square matrices $A_{1}, \ldots, A_{n}$ on its diagonal is denoted $\operatorname{diag}\left(A_{1}, \ldots, A_{n}\right)$.

For any matrix, $M, \mathbb{S}(M)$ is defined by $\mathbb{S}(M)=M+M^{T}$.

The smallest and largest eigenvalues of the matrix $M$ are respectively denoted $\lambda_{\min }(M)$ and $\lambda_{\max }(M)$.

The saturation function for a signal $v(t)$ is defined as

$$
\operatorname{sat}(v(t)):=\left\{\begin{array}{lll}
v(t) & \text { if } & v_{\min } \leq v(t) \leq v_{\max } \\
v_{\max } & \text { if } & v(t)>v_{\text {max }} \\
v_{\text {min }} & \text { if } & v(t)<v_{\text {min }}
\end{array}\right.
$$

where $v_{\max }$ and $v_{\min }$ denote the saturation levels.

\section{Problem STATEMENT}

\section{A. T-S modelling of the control saturation}

The main idea of this work is to model the nonlinear actuator saturation using the T-S representation (section IIA). For that, it is proposed to re-write the saturation equation (5) for each component of the control input vector under a particular form.

The $j^{t h}$ entry of the saturated control input, denoted $u_{s a t}^{j}(t)$, with the saturation levels $u_{\text {min }}^{j}$ and $u_{\text {max }}^{j}$ is written as

$$
u_{s a t}^{j}(t)=\sum_{i=1}^{3} \mu_{i}^{j}\left(u_{j}(t)\right)\left(\lambda_{i}^{j} u_{j}(t)+\gamma_{i}^{j}\right), \quad j=1, \ldots, n_{u}
$$

where $u_{j}(t)$ is the $j^{t h}$ component of $u(t)$, where

$$
\left\{\begin{array} { l } 
{ \lambda _ { 1 } ^ { j } = 0 } \\
{ \lambda _ { 2 } ^ { j } = 1 } \\
{ \lambda _ { 3 } ^ { j } = 0 }
\end{array} \quad \text { and } \left\{\begin{array}{l}
\gamma_{1}^{j}=u_{\min }^{j} \\
\gamma_{2}^{j}=0 \\
\gamma_{3}^{j}=u_{\max }^{j}
\end{array}\right.\right.
$$

and the weighting functions are defined

$$
\left\{\begin{array}{l}
\mu_{1}^{j}\left(u_{j}(t)\right)=\frac{1-\operatorname{sign}\left(u_{j}(t)-u_{\min }^{j}\right)}{2} \\
\mu_{2}^{j}\left(u_{j}(t)\right)=\frac{\operatorname{sign}\left(u_{j}(t)-u_{\min }^{j}\right)-\operatorname{sign}\left(u_{j}(t)-u_{\max }^{j}\right)}{2} \\
\mu_{3}^{j}\left(u_{j}(t)\right)=\frac{1+\operatorname{sign}\left(u_{j}(t)-u_{\max }^{j}\right)}{2}
\end{array}\right.
$$

Then, the control input vector $u(t) \in \mathbb{R}^{n_{u}}$ subject to actuator saturation is modeled by

$$
u_{\text {sat }}(t)=\left(\begin{array}{c}
\sum_{i=1}^{3} \mu_{i}^{1}\left(u_{1}(t)\right)\left(\lambda_{i}^{1} u_{1}(t)+\gamma_{i}^{1}\right) \\
\vdots \\
\sum_{i=1}^{3} \mu_{i}^{\ell}\left(u_{\ell}(t)\right)\left(\lambda_{i}^{\ell} u_{\ell}(t)+\gamma_{i}^{\ell}\right) \\
\vdots \\
\sum_{i=1}^{3} \mu_{i}^{n_{u}}\left(u_{n_{u}}(t)\right)\left(\lambda_{i}^{n_{u}} u_{n_{u}}(t)+\gamma_{i}^{n_{u}}\right)
\end{array}\right)
$$

From (9), one can notice that each input $u_{\ell}(t)$ has its own weighting function $\mu_{i}^{\ell}(t)$. In order that all the $n_{u}$ input vector components have the same weighting functions, based on the convex sum property (2), equation (9) can be written as

$$
u_{\text {sat }}(t)=\left(\begin{array}{c}
\sum_{i=1}^{3} \mu_{i}^{1}(t)\left(\lambda_{i}^{1} u_{1}(t)+\gamma_{i}^{1}\right)\left(\prod_{j=2}^{n_{u}} \sum_{i=1}^{3} \mu_{i}^{j}(t)\right) \\
\vdots \\
\sum_{i=1}^{3} \mu_{i}^{\ell}(t)\left(\lambda_{i}^{\ell} u_{\ell}(t)+\gamma_{i}^{\ell}\right)\left(\prod_{\substack{j=1 \\
j \neq \ell}}^{n_{u}} \sum_{i=1}^{3} \mu_{i}^{j}(t)\right) \\
\vdots \\
\sum_{i=1}^{3} \mu_{i}^{n_{u}}(t)\left(\lambda_{i}^{n_{u}} u_{n_{u}}(t)+\gamma_{i}^{n_{u}}\right)\left(\prod_{j=1}^{n_{u}-1} \sum_{i=1}^{3} \mu_{i}^{j}(t)\right)
\end{array}\right)
$$

For $n_{u}$ inputs, $3^{n_{u}}$ submodels are obtained. It is important to note that the actuator saturations $u_{\text {sat }}(t)$ are directly expressed in terms of the control variable $u(t)$ and its bounds $u_{\text {min }}^{j}=\gamma_{1}^{j}$ and $u_{\text {max }}^{j}=\gamma_{3}^{j}$.

Equation (10) is equivalent to

$$
u_{\text {sat }}(t)=\sum_{i=1}^{3^{n_{u}}} \mu_{i}^{s a t}(t)\left(\Lambda_{i} u(t)+\Gamma_{i}\right)
$$

The global weighting functions $\mu_{i}^{\text {sat }}(t)$, the matrices $\Lambda_{i} \in$ $\mathbb{R}^{n_{u} \times n_{u}}$ and vectors $\Gamma_{i} \in \mathbb{R}^{n_{u} \times 1}$ are defined as follows

$$
\left\{\begin{aligned}
\mu_{i}^{s a t}(t) & =\prod_{j=1}^{n_{u}} \mu_{\sigma_{i}^{j}}^{j}\left(u_{j}(t)\right) \\
\Lambda_{i} & =\operatorname{diag}\left(\lambda_{\sigma_{i}^{1}}^{1}, \ldots, \lambda_{\sigma_{i}^{n_{u}}}^{n_{u}}\right) \\
\Gamma_{i} & =\left[\gamma_{\sigma_{i}^{1}}^{1} \ldots \gamma_{\sigma_{i}^{n_{u}}}^{n_{n_{u}}}\right]^{T}
\end{aligned}\right.
$$

where the indexes $\sigma_{i}^{j}\left(i=1, \ldots, 3^{n_{u}}\right.$ and $\left.j=1, \ldots, n_{u}\right)$, equal to 1,2 or 3 , indicate which partition of the $j^{\text {th }}$ input $\left(\mu_{1}^{j}, \mu_{2}^{j}\right.$ or $\mu_{3}^{j}$ ) is involved in the $i^{\text {th }}$ submodel.

The relations between the $i^{\text {th }}$ submodel and the $\sigma_{i}^{j}$ indices are given by the following equation

$$
i=3^{n_{u}-1} \sigma_{i}^{1}+3^{n_{u}-2} \sigma_{i}^{2}+\ldots+3^{0} \sigma_{i}^{n_{u}}-\left(3^{1}+3^{2}+\ldots+3^{n_{u}-1}\right)
$$

The $\sigma_{i}^{j}$ are such that $\left(\left(\sigma_{i}^{1}-1\right), \ldots,\left(\sigma_{i}^{n_{u}}-1\right)\right)$ corresponds to (i-1) in base 3 . For more details, see [1]. 


\section{B. Uncertain saturated system description}

Let us now consider a T-S uncertain nonlinear system with input saturation represented by the following state equation

$$
\dot{x}(t)=\sum_{i=1}^{n} \mu_{i}(\xi(t))\left(\left(A_{i}+\Delta A(t)\right) x(t)+\left(B_{i}+\Delta B(t)\right) u_{s a t}(t)\right)
$$

where

$$
\begin{aligned}
& \Delta A(t)=A \Sigma_{A}(t) E_{A} \\
& \Delta B(t)=B \Sigma_{B}(t) E_{B}
\end{aligned}
$$

with

$$
\begin{aligned}
& \Sigma_{A}^{T}(t) \Sigma_{A}(t) \leq I, \quad \forall t \\
& \Sigma_{B}^{T}(t) \Sigma_{B}(t) \leq I, \quad \forall t
\end{aligned}
$$

$I$ being the identity matrix, $A, B, E_{A}$ and $E_{B}$ matrices of appropriate dimensions.

Using (11), equation (13) can be written as

$$
\begin{aligned}
\dot{x}(t)=\sum_{i=1}^{n} \sum_{k=1}^{3^{n_{u}}} \mu_{i}(\xi(t)) \mu_{k}^{s a t}(t)\left(\left(A_{i}+\Delta A(t)\right) x(t)\right. \\
\left.+\left(B_{i}+\Delta B(t)\right)\left(\Lambda_{k} u(t)+\Gamma_{k}\right)\right)
\end{aligned}
$$

\section{SATURATEd STATE FEedBACK CONTROL INPUT}

The objective is to design a stabilizing time-varying state feedback controller ensuring the stability of the system, even in the presence of control input saturation and uncertainties. The solution is obtained by representing the saturation as a T-S system and by solving an optimization problem under LMI constraints.

\section{A. Nominal control law (without saturation)}

In this section, a nonlinear state feedback controller sharing the same weighting functions as those of the T-S model is designed. Since it is the nominal case, the controller gains are synthesized without taking into account the saturation limits.

Let us consider the following unsaturated control adopted for stabilizing the system to the origin

$$
u(t)=-\sum_{j=1}^{n} \mu_{j}(\xi(t)) K_{j} x(t)
$$

where the matrices $K_{j} \in \mathbb{R}^{n_{u} \times n_{x}}$ are the controller gains to be determined.

Replacing (19) in equation (13) without input saturation, it becomes

$$
\begin{aligned}
& \dot{x}(t)=\sum_{i=1}^{n} \sum_{j=1}^{n} \mu_{i}(\xi(t)) \mu_{j}(\xi(t))\left(A_{i}-B_{i} K_{j}+\right. \\
&\left.\Delta A(t)-\Delta B(t) K_{j}\right) x(t)
\end{aligned}
$$

In order to study the stability of (20), the following Lyapunov function is defined

$$
V(x(t))=x^{T}(t) P x(t)
$$

where $P \in \mathbb{R}^{n_{x}}$ is a symmetric positive definite matrix.

To ensure the stability of (20), the conditions to satisfy are the following (see [4] for the proof)

$$
\left(\begin{array}{ccccc}
\mathbb{S}\left(A_{i} P_{1}-B_{i} R_{j}\right) & P_{1} E_{A}^{T} & \omega_{1} A & R_{j}^{T} E_{B}^{T} & \omega_{2} B \\
* & -\omega_{1} I & 0 & 0 & 0 \\
* & * & -\omega_{1} I & 0 & 0 \\
* & * & * & -\omega_{2} I & 0 \\
* & * & * & * & -\omega_{2} I
\end{array}\right)<0
$$

with $P_{1}=P^{-1}, \omega_{1}, \omega_{2}$ positive scalars. The controller gains $K_{j}$ in (19) are computed by: $K_{j}=R_{j} P_{1}^{-1}$, for $i, j=1, \ldots, n$.

\section{B. Controller with saturation constraint}

In this section, our objective is to design a time-varying state feedback controller (19) which gains depend on the saturation limits to guarantee the stability of the uncertain system (18) despite of the uncertainties and of the saturated input.

By replacing the control law (19) in the T-S system equation (18), the obtained system is the following

$$
\begin{aligned}
\dot{x}(t)= & \sum_{i=1}^{n} \sum_{j=1}^{n} \sum_{k=1}^{3^{n u}} \mu_{i}(\xi(t)) \mu_{j}(\xi(t)) \mu_{k}^{s a t}(t)\left(\left(A_{i}-B_{i} \Lambda_{k} K_{j}\right) x(t)\right. \\
& \left.+\left(\Delta A(t)-\Delta B(t) \Lambda_{k} K_{j}\right) x(t)+B_{i} \Gamma_{k}+\Delta B(t) \Gamma_{k}\right)
\end{aligned}
$$

Theorem 1: There exists a time-varying state feedback controller (19) for a saturated input uncertain system (18) ensuring that the system state converges toward an origincentred ball of radius bounded by $\beta>0$, if there exists matrices $P_{1}=P_{1}^{T}>0, R_{j}, \Sigma_{k}=\Sigma_{k}^{T}>0$, and positive scalars $\sigma_{k}, \omega_{1}, \omega_{2 k}$ solutions of the following optimization problem

$$
\min _{P_{1}, R_{j}, \Sigma_{k}, \sigma_{k}, \omega_{1}, \omega_{2 k}} \beta
$$

s.t.

$$
\left(\begin{array}{cc}
Q_{i j k} & I \\
I & -\beta I
\end{array}\right)<0
$$

with $Q_{i j k}$ defined by (27), for $i=1, \ldots, n, j=1, \ldots, n$ and $k=1, \ldots, 3^{n_{u}}$ (see next page).

$$
\Gamma_{k}^{T} B_{i}^{T} \Sigma_{k} B_{i} \Gamma_{k}+\sigma_{k} \Gamma_{k}^{T} E_{B}^{T} E_{B} \Gamma_{k}<\beta
$$

The gains of the controller are given by

$$
K_{j}=R_{j} P_{1}^{-1}
$$

Proof: According to state equations (23), the time derivative of the Lyapunov function (21) is given by

$$
\begin{gathered}
\dot{V}(x(t))=\sum_{i=1}^{n} \sum_{j=1}^{n} \sum_{k=1}^{3^{n_{u}}} \mu_{i}(\xi) \mu_{j}(\xi) \mu_{k}^{s a t}(t)\left(x^{T}(t) P B_{i} \Gamma_{k}+\right. \\
\Gamma_{k}^{T} B_{i}^{T} P x(t)+x^{T}(t) P \Delta B(t) \Gamma_{k}+\Gamma_{k}^{T} \Delta B^{T}(t) P x(t)+ \\
x^{T}(t)\left(\left(A_{i}-B_{i} \Lambda_{k} K_{j}\right)^{T} P+P\left(A_{i}-B_{i} \Lambda_{k} K_{j}\right)+\right. \\
\left.\left.\left(\Delta A(t)-\Delta B(t) \Lambda_{k} K_{j}\right)^{T} P+P\left(\Delta A(t)-\Delta B(t) \Lambda_{k} K_{j}\right)\right) x(t)\right)
\end{gathered}
$$

Using Lemma 3, it follows that for $\Sigma_{k}=\Sigma_{k}^{T}>0$

$$
x^{T}(t) P B_{i} \Gamma_{k}+\Gamma_{k}^{T} B_{i}^{T} P x(t) \leq \Gamma_{k}^{T} B_{i}^{T} \Sigma_{k} B_{i} \Gamma_{k}+x^{T}(t) P \Sigma_{k}^{-1} P x(t)
$$




$$
Q_{i j k}=\left(\begin{array}{ccccc}
P_{1} A_{i}^{T}+A_{i} P_{1}-R_{j}^{T} \Lambda_{k}^{T} B_{i}^{T}-B_{i} \Lambda_{k} R_{j}+\omega_{1} A A^{T}+\omega_{2 k} B B^{T} & I & B & P_{1} E_{A}^{T} & R_{j}^{T} \Lambda_{k}^{T} E_{B}^{T} \\
* & -\Sigma_{k} & 0 & 0 & 0 \\
* & * & -\sigma_{k} I & 0 & 0 \\
* & * & * & -\omega_{1} I & 0 \\
* & * & * & * & -\omega_{2 k} I
\end{array}\right)
$$

Using Lemma 3 and definition (15), it follows that, for any positive scalar $\sigma_{k}$, it holds

$$
\begin{aligned}
x^{T}(t) P \Delta B(t) \Gamma_{k}+ & \Gamma_{k}^{T} \Delta B(t)^{T} P x(t) \leq \\
& \sigma_{k} \Gamma_{k}^{T} E_{B}^{T} E_{B} \Gamma_{k}+\sigma_{k}^{-1} x^{T}(t) P B B^{T} P x(t)
\end{aligned}
$$

From (30) and (31), the time derivative of the Lyapunov function (29) is bounded as follows

$$
\begin{array}{r}
\dot{V}(x(t)) \leq \sum_{i=1}^{n} \sum_{j=1}^{n} \sum_{k=1}^{3^{n_{u}}} \mu_{i}(\xi) \mu_{j}(\xi) \mu_{k}^{s a t}(t)\left(\Gamma_{k}^{T} B_{i}^{T} \Sigma_{k} B_{i} \Gamma_{k}+\right. \\
\left.\sigma_{k} \Gamma_{k}^{T} E_{B}^{T} E_{B} \Gamma_{k}+x^{T}(t) \mathscr{Q}_{i j k} x(t)\right)
\end{array}
$$

where

$$
\begin{gathered}
\mathscr{Q}_{i j k}=\left(A_{i}-B_{i} \Lambda_{k} K_{j}\right)^{T} P+P\left(A_{i}-B_{i} \Lambda_{k} K_{j}\right)+\sigma_{k}^{-1} P B B^{T} P \\
+\left(\Delta A(t)-\Delta B(t) \Lambda_{k} K_{j}\right)^{T} P+P\left(\Delta A(t)-\Delta B(t) \Lambda_{k} K_{j}\right)+P \Sigma_{k}^{-1} P
\end{gathered}
$$

Applying Lemma 2 with $Y=P_{1}$ and the following variable changes

$$
\left\{\begin{array}{l}
P_{1}=P^{-1} \\
R_{j}=K_{j} P_{1}
\end{array}\right.
$$

the inequality $\mathscr{Q}_{i j k}<0$ is equivalent to

$$
\begin{gathered}
P_{1} A_{i}^{T}+A_{i} P_{1}-R_{j}^{T} \Lambda_{k}^{T} B_{i}^{T}-B_{i} \Lambda_{k} R_{j}+\Sigma_{k}^{-1}+\sigma_{k}^{-1} B B^{T}+ \\
P_{1} \Delta A^{T}(t)+\Delta A(t) P_{1}-R_{j}^{T} \Lambda_{k}^{T} \Delta B^{T}(t)-\Delta B(t) \Lambda_{k} R_{j}<0
\end{gathered}
$$

From (14-17), with Lemma 3, it follows

$$
\begin{gathered}
P_{1} \Delta A^{T}(t)+\Delta A(t) P_{1} \leq \omega_{1}^{-1} P_{1} E_{A}^{T} E_{A} P_{1}+\omega_{1} A A^{T} \\
-R_{j}^{T} \Lambda_{k}^{T} \Delta B^{T}(t)-\Delta B(t) \Lambda_{k} R_{j} \leq \\
\omega_{2 k}^{-1} R_{j}^{T} \Lambda_{k}^{T} E_{B}^{T} E_{B} \Lambda_{k} R_{j}+\omega_{2 k} B B^{T}
\end{gathered}
$$

where $\omega_{1}$ and $\omega_{2 k}$ are positive scalars.

From (36) and (37), $\mathscr{Q}_{i j k}<0$ is satisfied if

$$
\begin{gathered}
P_{1} A_{i}^{T}+A_{i} P_{1}-R_{j}^{T} \Lambda_{k}^{T} B_{i}^{T}-B_{i} \Lambda_{k} R_{j}+\Sigma_{k}^{-1}+\sigma_{k}^{-1} B B^{T}+ \\
\omega_{1}^{-1} P_{1} E_{A}^{T} E_{A} P_{1}+\omega_{1} A A^{T}+\omega_{2 k}^{-1} R_{j}^{T} \Lambda_{k}^{T} E_{B}^{T} E_{B} \Lambda_{k} R_{j}+\omega_{2 k} B B^{T}<0
\end{gathered}
$$

Applying Schur's complement, (38) is equivalent to $Q_{i j k}<0$ which is implied by (25).

In order to analyse (32), let us now define

$$
\begin{gathered}
\varepsilon=\min _{i=1: n, j=1: n, k=1: 3^{n_{u}}} \lambda_{\min }\left(-Q_{i j k}\right) \\
\delta=\max _{i=1: n, k=1: 3^{n_{u}}} \Gamma_{k}^{T} B_{i}^{T} \Sigma_{k} B_{i} \Gamma_{k}+\sigma_{k} \Gamma_{k}^{T} E_{B}^{T} E_{B} \Gamma_{k}
\end{gathered}
$$

Since $\Sigma_{k}, \sigma_{k}>0$, from (32), it follows that $\dot{V}(t)<-\varepsilon\|x\|^{2}$ $+\delta$. Then $\dot{V}(t)<0$ holds if

$$
\left\{\begin{array}{r}
\mathscr{Q}_{i j k}<0 \\
\|x\|^{2}>\frac{\delta}{\varepsilon}
\end{array}\right.
$$

According to Lyapunov theory [10], it means that $x(t)$ is uniformly bounded and converges to an origin-centred ball of radius $\sqrt{\frac{\delta}{\varepsilon}}$.

Since $\mathscr{Q}_{i j k}<0$ is ensured by (25), the objective is now to minimize the radius $\sqrt{\frac{\delta}{\varepsilon}}$. Firstly $\delta$ is bounded by $\beta$ from the definition (40) and the LMIs (26). From (25), with a Schur complement, it obviously follows that

$$
(1 / \beta) I<-Q_{i j k}, i=1, \ldots, n, j=1, \ldots, n, k=1, \ldots, 3^{n_{u}}
$$

implying that all the eigenvalues of $\left(-Q_{i j k}\right)$ are larger that $1 / \beta$. As a consequence $1 / \beta<\varepsilon$ holds, and finally the radius is bounded by $\beta$. The minimal value of $\beta$ is obtained from (24).

\section{SATURATED STATE FEEDBACK CONTROL INPUT : A DESCRIPTOR APPROACH}

In this section, in order to reduce the number of LMIs to be solved, the descriptor approach is applied. This approach is well known to avoid the coupling terms between the feedback gains and the Lyapunov matrices. As a consequence, the number of LMIs decreases and relaxed conditions are obtained [3].

The control law given by (19) is written as follows

$$
0 . \dot{u}(t)=-\sum_{j=1}^{n} \mu_{j}(\xi(t)) K_{j} x(t)-u(t)
$$

Let us consider the augmented state vector $x_{a}^{T}(t)=$ $\left(\begin{array}{ll}x^{T}(t) & u^{T}(t)\end{array}\right)^{T}$. From equations (18) and (43), it follows

$$
E \dot{x}_{a}(t)=\sum_{i=1}^{n} \sum_{k=1}^{3^{n_{u}}} \mu_{i}(\xi(t)) \mu_{k}^{s a t}(t)\left(\mathscr{A}_{i k}(t) x_{a}(t)+\mathscr{B}_{i k}(t)\right)
$$

with

$$
\begin{gathered}
E=\operatorname{diag}(I, 0) \\
\mathscr{A}_{i k}(t)=\left(\begin{array}{cc}
A_{i}+\Delta A(t) & B_{i} \Lambda_{k}+\Delta B(t) \Lambda_{k} \\
-K_{i} & -I
\end{array}\right) \\
\mathscr{B}_{i k}(t)=\mathscr{B}_{i k}^{1}+\mathscr{B}_{i k}^{2}(t) \\
\mathscr{B}_{i k}^{1}=\left(\begin{array}{c}
B_{i} \Gamma_{k} \\
0
\end{array}\right), \mathscr{B}_{i k}^{2}(t)=\left(\begin{array}{c}
\Delta B(t) \Gamma_{k} \\
0
\end{array}\right)
\end{gathered}
$$

Theorem 2: There exists a time-varying state feedback controller (19) for a saturated input uncertain T-S system (18) 
ensuring that the system state converges toward an origincentred ball of radius bounded by $\beta$, if there exists matrices $P_{1}=P_{1}^{T}>0, P_{2}>0, R_{i}, \Sigma_{k}=\Sigma_{k}^{T}>0$, and positive scalars $\lambda_{1 k}, \lambda_{2}, \lambda_{3}$ solutions of the following optimization problem

$$
\min _{P_{1}, P_{2}, R_{i}, \Sigma_{k}, \lambda_{1 k}, \lambda_{2}, \lambda_{3}} \beta
$$

s.t.

$$
\left(\begin{array}{cc}
\Xi_{i k} & I \\
I & -\beta I
\end{array}\right)<0
$$

with $\Xi_{i k}$ defined by (51), for $i=1, \ldots, n$ and $k=1, \ldots, 3^{n_{u}}$ (see next page) and

$$
\Gamma_{k}^{T} \Sigma_{k} \Gamma_{k}+\lambda_{1 k} \Gamma_{k}^{T} E_{B}^{T} E_{B} \Gamma_{k}<\beta
$$

The gains of the controller are given by

$$
K_{i}=P_{2}^{-1} R_{i}
$$

Proof: Let us consider the following Lyapunov function

$$
V(t)=x_{a}^{T}(t) E^{T} P x_{a}(t)
$$

with the condition $E^{T} P=P^{T} E \geq 0$. The matrix $P$ is chosen $P=\operatorname{diag}\left(P_{1}, P_{2}\right)$, with $P_{1}=P_{1}^{T}>0$.

The time derivative of the Lyapunov function is given by

$$
\begin{aligned}
\dot{V}(t)=\sum_{i=1}^{n} \sum_{k=1}^{3^{n_{u}}} \mu_{i}( & \xi(t)) \mu_{k}^{s a t}(t)\left(\mathscr{B}_{i k}^{T}(t) P x_{a}(t)+x_{a}^{T}(t) P^{T} \mathscr{B}_{i k}(t)\right. \\
& +x_{a}^{T}(t)\left(\mathscr{A}_{i k}^{T}(t) P+P^{T} \mathscr{A}_{i k}(t)\right) x_{a}(t)
\end{aligned}
$$

The main idea for the proof is to separate the constant and the time-varying parts in $\mathscr{A}_{i k}(t)$ and $\mathscr{B}_{i k}(t)$. Then, based on properties (16) and (17), the time-varying part is bounded. Using lemma 1 and 3 with equations (14) and (15), for any symmetric positive definite matrices $\Sigma_{k}$, positive scalars $\lambda_{1 k}$ and positive scalars $\lambda_{2}$ and $\lambda_{3}$, it follows

$$
\begin{gathered}
x_{a}^{T}(t) P^{T} \mathscr{B}_{i k}^{1}+\left(\mathscr{B}_{i k}^{1}\right)^{T} P x_{a}(t) \leq \\
\Gamma_{k}^{T} \Sigma_{k} \Gamma_{k}+x^{T}(t) P_{1} B_{i} \Sigma_{k}^{-1} B_{i}^{T} P_{1} x(t) \\
\left(\mathscr{B}_{i k}^{2}\right)^{T}(t) P x_{a}(t)+x_{a}^{T}(t) P^{T} \mathscr{B}_{i k}^{2}(t) \leq \\
\lambda_{1 k} \Gamma_{k}^{T} E_{B}^{T} E_{B} \Gamma_{k}+\left(\lambda_{1 k}\right)^{-1} x^{T}(t) P_{1} B B^{T} P_{1} x(t) \\
x^{T}(t) P_{1} \Delta A(t) x(t)+x^{T}(t) \Delta A^{T}(t) P_{1} x(t) \\
\leq \lambda_{2} x^{T}(t) E_{A}^{T} E_{A} x(t)+\lambda_{2}^{-1} x^{T}(t) P_{1} A A^{T} P_{1} x(t) \\
x^{T}(t) P_{1} \Delta B(t) \Lambda_{k} u(t)+u^{T}(t) \Lambda_{k}^{T} \Delta B^{T}(t) P_{1} x(t) \\
\leq \lambda_{3}^{-1} x^{T}(t) P_{1} B B^{T} P_{1} x(t)+\lambda_{3} u^{T}(t) \Lambda_{k}^{T} E_{B}^{T} E_{B} \Lambda_{k} u(t)
\end{gathered}
$$

The time derivative $\dot{V}(t)$ (55) is then bounded by

$$
\begin{aligned}
\dot{V}(t) \leq \sum_{i=1}^{n} \sum_{k=1}^{3^{n_{u}}} \mu_{i}(\xi(t)) \mu_{k}^{s a t}(t)\left(x_{a}^{T}(t) \mathscr{M}_{i k} x_{a}(t)\right. \\
\left.+\Gamma_{k}^{T} \Sigma_{k}^{1} \Gamma_{k}+\sigma_{k}^{2} \Gamma_{k}^{T} E_{B}^{T} E_{B} \Gamma_{k}\right)
\end{aligned}
$$

with

$$
\begin{gathered}
\mathscr{M}_{i k}=\left(\begin{array}{cc}
\mathscr{M}_{i k}^{1} & -K_{i}^{T} P_{2}+P_{1} B_{i} \Lambda_{k} \\
* & -P_{2}-P_{2}^{T}+\omega_{2} \Lambda_{k}^{T} E_{B}^{T} E_{B} \Lambda_{k}
\end{array}\right) \\
\mathscr{M}_{i k}^{1}=A_{i}^{T} P_{1}+P_{1} A_{i}+\lambda_{2} E_{A}^{T} E_{A}+\lambda_{2}^{-1} P_{1} A A^{T} P_{1} \\
+\left(\lambda_{1 k}\right)^{-1} P_{1} B B^{T} P_{1}+P_{1} B \Sigma_{k}^{-1} B^{T} P_{1}+\lambda_{3}^{-1} P_{1} B B^{T} P_{1}
\end{gathered}
$$

Applying Schur's complement, $\dot{V}(t)<0$ holds if $\Xi_{i k}<0$ and $\|x\|^{2}>\frac{\delta}{\varepsilon} . \Xi_{i k}<0$ is ensured from (50). The minimization of the upper bound value $\beta$ is done as the previous case.

\section{NUMERICAL EXAMPLE}

The proposed state feedback controller design for systems with saturated control input is illustrated by the following academic example. Let consider the uncertain nonlinear system (13) with $n=2$ and

$$
\begin{aligned}
& A_{1}=\left(\begin{array}{cc}
-1 & 1 \\
0 & -0.75
\end{array}\right), \quad A_{2}=\left(\begin{array}{cc}
-0.80 & 0.02 \\
0.20 & -1.40
\end{array}\right) \\
& A=\left(\begin{array}{cc}
0.1 & 1 \\
1 & 0.1
\end{array}\right), \quad E_{A}=\left(\begin{array}{cc}
0.2 & 1 \\
1 & 0.5
\end{array}\right) \\
& B_{1}=\left(\begin{array}{ll}
2 & 2 \\
2 & 2
\end{array}\right), \quad B_{2}=\left(\begin{array}{cc}
0.75 & 0 \\
-0.5 & 0.75
\end{array}\right) \\
& B=\left(\begin{array}{cc}
0 & 0.1 \\
0.1 & 0
\end{array}\right), \quad E_{B}=\left(\begin{array}{cc}
0.1 & 0.1 \\
0.1 & 0.1
\end{array}\right)
\end{aligned}
$$

$\Sigma_{A}(t)=\Sigma_{B}(t)=\sigma(t) I$ with $\sigma(t)$ depicted in Fig. 1 The input

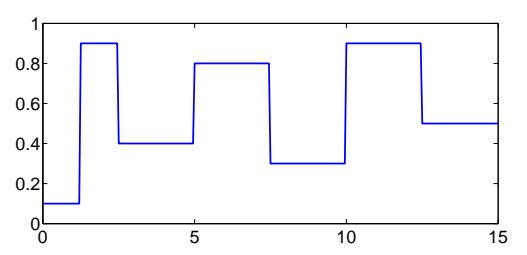

Fig. 1. Uncertainty $\sigma(t)$

vector is subject to the following actuator saturations

$$
u_{1 \max }=u_{2 \max }=2 \quad, \quad u_{1 \min }=u_{2 \min }=-2
$$

The weighting functions are defined as follows

$\mu_{1}(x(t))=\frac{\left(1-\tanh \left(x_{1}(t)+x_{2}(t)\right)\right)}{2} ; \mu_{2}(x(t))=1-\mu_{1}(x(t))$

In order to illustrate the effectiveness of the proposed approach, a so-called nominal controller is computed without taking into account the input saturation, although the saturation acts on the control input. Then a comparison is provided between the nominal closed loop system without saturation, the nominal closed loop system with saturated actuators and the closed loop system with the controller proposed in this paper where the controller design depends on the saturation bounds.

For the considered example, the calculated nominal gains are

$$
K_{n 1}=\left(\begin{array}{ll}
1.10 & 1.11 \\
0.93 & 0.94
\end{array}\right), K_{n 2}=\left(\begin{array}{ll}
1.64 & 1.64 \\
1.39 & 1.39
\end{array}\right)
$$

The control gains $K_{1}, K_{2}$ computed from theorem 1 are

$$
K_{1}=\left(\begin{array}{cc}
9.73 & 8.97 \\
11.52 & 10.72
\end{array}\right), K_{2}=\left(\begin{array}{cc}
9.73 & 8.97 \\
11.52 & 10.72
\end{array}\right)
$$

Applying the descriptor approach, the controller gains are

$$
K_{1 d}=\left(\begin{array}{cc}
0.28 & 0.02 \\
0.062 & 0.42
\end{array}\right), K_{2 d}=\left(\begin{array}{cc}
0.58 & 0.36 \\
0.37 & 0.61
\end{array}\right)
$$




$$
\Xi_{i k}=\left(\begin{array}{cccccc}
A_{i}^{T} P_{1}+P_{1} A_{i}+\lambda_{2} E_{A}^{T} E_{A} & -R_{i}+P_{1} B_{i} \Lambda_{k} & P_{1} A & P_{1} B & P_{1} B & P_{1} B \\
* & -P_{2}-P_{2}^{T}+\lambda_{3} \Lambda_{k}^{T} E_{B}^{T} E_{B} \Lambda_{k} & 0 & 0 & 0 & 0 \\
* & * & -\lambda_{2} I & 0 & 0 & 0 \\
* & * & * & -\lambda_{1 k} I & 0 & 0 \\
* & * & * & * & -\Sigma_{k} & 0 \\
* & * & * & * & * & -\lambda_{3} I
\end{array}\right)<0
$$
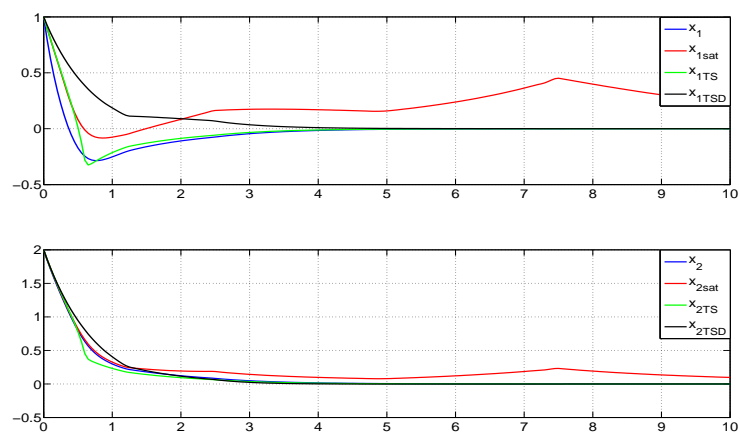

Fig. 2. Time evolution of system states
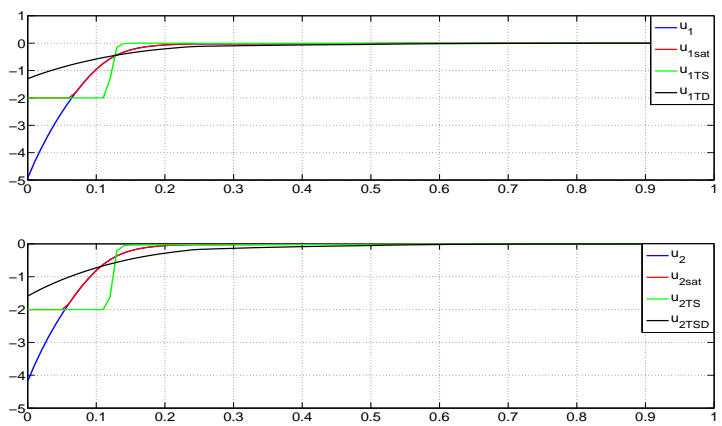

Fig. 3. Control inputs

On the figures 2 and 3, are depicted the system states and control inputs of the nominal closed loop system without saturation (respectively denoted $x_{1}, x_{2}, u_{1}$ and $u_{2}$ ), those of the nominal closed loop system with saturation (respectively denoted $x_{1 s a t}, x_{2 s a t}, u_{1 s a t}$ and $\left.u_{2 s a t}\right)$ and those of the proposed approach (respectively denoted $x_{1 T S}, x_{2 T S}, u_{1 T S}$ and $u_{2 T S}$ ) and $\left(x_{1 T S D}, x_{2 T S D}, u_{1 T S D}\right.$ and $\left.u_{2 T S D}\right)$ for the descriptor approach.

One can observe from the depicted figures that the input saturation has a destabilizing effect if it is not taken into account in the controller design. In the other hand, for the proposed T-S approach, both stabilization and state trajectory convergence to the origin are ensured in spite of the input saturation and modelling uncertainities. Theorem 2 gives the stability conditions obtained with a descriptor approach. The number of LMIs $\left(n \times 3^{n_{u}}\right)$ is less than those of theorem 1 $\left(n^{2} \times 3^{n_{u}}\right)$ and the obtained results are slightly better. We note that the fall time is almost the same $(2 \mathrm{~s})$, with a state converging toward an origin-centered ball of radius equal to
4.20 for the first approach and 3.24 for the second one.

\section{CONCLUSIONS AND FUTURE WORKS}

An uncertain nonlinear system with saturated control input can be represented by a Takagi-Sugeno model, including the input saturation. This unified representation allows to simultaneously deal with these difficulties and to synthesize a PDC controller which gains depend on the saturation bounds. The solution of this problem is based on the Lyapunov theory and expressed in terms of LMI. The descriptor approach is also proposed, allowing to divide the number of LMI to solve by $n$, the number of subsystems. It is important to highlight that the main advantage of the proposed approach is the stability ensurance of the saturated uncertain nonlinear systems. A numerical example is presented in order to illustrate the proposed approach. The provided example shows that the proposed controller is able to conteract the destabilizing effect of the saturation affecting the control input.

\section{REFERENCES}

[1] S. Bezzaoucha, B. Marx, D. Maquin, and J. Ragot. Linear feedback control input under actuator saturation : A Takagi-Sugeno approach. In $2^{\text {nd }}$ International Conference on Systems and Control (ICSC'12), Marrakech, Morocco, June 20-22 2012.

[2] Y-Y. Cao and Z. Lin. Robust stability analysis and fuzzy-scheduling control for nonlinear systems subject to actuator saturation. IEEE Transactions on Fuzzy Systems, 11(1):57-67, 2003.

[3] K. Guelton, T. Bouarar, and N. Manamanni. Robust dynamic output feedback fuzzy Lyapunov stabilization of Takagi-Sugeno systems- a descriptor redundancy approach. Fuzzy Sets and Systems, 160(19):2796-2811, 2009.

[4] D. Ichalal, B. Marx, J. Ragot, and D. Maquin. Robust observer design for uncertain Takagi-Sugeno model with unmeasurable decision variables: an $L_{2}$ approach. In $16^{\text {th }}$ Mediterranean Conference on Control and Automation, MED, Ajaccio, Corsica, France, June 25272008.

[5] Z. Lin and A. Saberi. Semi-global exponential stabilization of linear systems subject to input saturation via linear feedbacks. System and Control Letters, 21(3):225-239, 1993.

[6] A. Syaichu-Rohman. Optimisation Based Feedback Control of Input Constrained Linear Systems. PhD thesis, The University of Newcastle Callaghan, Australia, 2005.

[7] A. Syaichu-Rohman and R.H. Middleton. Anti-windup schemes for discrete time systems: An LMI-based design. In $5^{\text {th }}$ Asian Control Conference, Australia, 20-23 July 2004.

[8] K. Tanaka and H.O. Wang. Fuzzy Control Systems Design and Analysis: A Linear Matrix Inequality Approach. John Wiley \& Sons, Inc., 2001.

[9] S. Tarbouriech, G. Garcia, J.M. Gomes da Silva Jr., and I. Queinnec. Stability and Stabilization of Linear Systems with Saturating Actuators. Springer-Verlag, 2011.

[10] K. Zhang, B. Jiang, and P. Shi. A new approach to observer-based fault-tolerant controller design for Takagi-Sugeno fuzzy systems with state delay. Circuits, systems, and signal processing, 28(5):679-697, 2009.

[11] B. Zhou, G. Duan, and Z. Lin. A parametric Lyapunov equation approach to the design of low gain feedback. IEEE Transactions on Automatic Control, 53(6):1548-1554, 2008. 